\title{
Spectrum Sharing Technique for Cooperative Cognitive Radio Networks using Bipartite Graph
}

\author{
Ashish Kumar Sinha \\ Research Scholar \\ Department of \\ Electronics and \\ Communication Engineering, \\ NIIST, Bhopal, M.P
}

\author{
Rajeev Thakur \\ Associate Professor \\ Department of \\ Electronics and \\ Communication Engineering, \\ NIIST, Bhopal, M.P
}

\begin{abstract}
This paper presents, a spectrum sharing strategy in cooperative cognitive radio network (CCRN). A multi-phase cooperation architecture is explained and studied with cooperation partner selection and spectrum sharing among secondary users (SUs).The data of primary users (PUs) forwarded to the cooperation partners who are selected from SUs, and then acquire the spectrum access opportunities for their own transmissions as a reward. The partner selection is modeled as an optimally weighted bipartite matching problem to maximize the total utility where energy efficiency is also considered just to increase the utility for the PU-SU cooperation pairs. By the partner SU further improvisation in the spectrum utilization is done by sharing the acquired spectrum with the surrounding SUs via cooperative network coding. At the end the simulation results provided, which shows that to the dynamic traffic loads in CCRN, the proposed partner selection and spectrum sharing approach adapts well.
\end{abstract}

\section{Keywords}

CCRN, QoS, IUs, MMSE

\section{INTRODUCTION}

The scarcity of spectral resources has become a severe problem due to the significant growth in commercial wireless services, in recent years, with the emergence of cooperative communications in wireless networks [3], a new communication paradigm in cooperative cognitive radio networks is proposed [4-6], termed cooperative cognitive radio networks (CCRN). The traditional fixed spectrum allocation is proved inefficient, since the frequency band is largely under-utilized [1]. Cognitive Radio (CR) [2] has been considered as a promising technology for improve spectrum utilization by allowing secondary users (SUs) to access spectrum holes unoccupied by primary users (Pus).

The rapid growth in wireless communications has contributed a huge demand on the deployment of new wireless services in both the licensed and unlicensed frequency spectrum. However, recent Studies show the fixed spectrum assignment policy enforced today results in poor spectrum utilization. To address this problem, cognitive radio [1,2] has emerged as a promising technology to enable the access of the intermittent periods of unoccupied frequency bands, known as white space or spectrum holes, and thereby increase the spectral efficiency.

The fundamental task of each Cognitive radio user in cognitive radio networks, in themost primitive sense, for detection of licensed users, also called as primary users (PUs), if they are present and identify the available spectrum if they are absent. This is usually achieved by sensing the RF environment, process called spectrum sensing [1-4]. The objectives of spectrum sensing are twofold: first, CR users should not cause harmful interference to PUs by either switching to an available band or limiting its interference with PUs at an acceptable level and, second, CR users should efficiently identify the spectrum holes for required throughput and quality-of service(QoS). Thus, the detection performance in spectrum sensing is very much crucial to the performance of both primary and CR networks.

In the conventional CCRN formulation, some type of resource allocation problem was addressed, such as subchannel assignment for SUs, relay assignment, and power control [46]. In [4], the subcarrier assignment, relay assignment, and SU relay strategy optimization problems were approached with flexible channel cooperation in a multi-channel CCRN, where a unified optimization framework based on Nash Bargaining Solutions was developed. In $[5,6]$, the spectrum leasing problem was formulated for one PU and multiple SUs as a Stackelberg game and the Nash equilibrium was derived. A single channel was assumed available, and different transmissions were divided in time. The consideration of one channel and one PU in [5,6] presents a simplification for practical scenarios where there are typically multiple channels and multiple PUs that coexist in the coverage area of a base station in the cellular network.

A multiphase cooperation scheme is proposed in order to improve the network utility as well as the spectrum access opportunity. We assign the selected relaying SUs as the group of intermediate users (IUs), which cooperate with PUs in traffic relay and share the spectrum access opportunities with the remaining SUs, respectively. With the help of IUs, the PUs can improve their own performance as well as not be involved in such a complicated cooperation scheme with multiple SUs. Meanwhile, the SUs starving for the spectrum access opportunities attain what they want as well.

Second, an IU selection scheme is implemented by the maximum weighted bipartite matching algorithm, and the utility of the cooperating pairs is enhanced by exploiting the ratio of cooperation pairs' utility to the total energy consumption with the consideration of the IUs' energy efficiency. Third, through the cooperation among the IUs and the surrounding SUs by using cooperative network coding, the starving SUs who form a cluster can obtain the transmission opportunities without consuming too much energy to relay the PUs' traffic. Conversely, the IUs' utility and communication reliability can be enhanced. 


\section{SYSTEM MODEL}

As demonstrated in Fig., we consider PUs and SUs are uniformly distributed in a CCRN. The data has been transmitted to the BS over its own licensed channel by a base station (BS) serves $\square$ PUs and each PU, given that the spectrums of PUs are orthogonal in frequency and/or space. access points (APs) coexist in the same area serving $\square$ SUs and each SU communicates with its corresponding AP.

The first phase cooperation is between the PU and the selected cluster head IU, while the second phase cooperation is between the cluster head and other SUs in the cluster. As shown in Fig. 2, the cooperation between SUs and Pus takes place in a two-phase cooperation scheme in each time slot $\square$. The partner IU selection scheme is first performed, and then the cluster head IU cooperates with the PU in a TDMA manner that the PU transmits its package to the cooperating IU and the IU relays PU's last package to the BS simultaneously. After the cooperation between PU and IU, the IU finds the cooperative SUs who form a cluster from the surrounding starving SUs. Then, the IU and the SUs in the cluster cooperate by cooperative network coding.

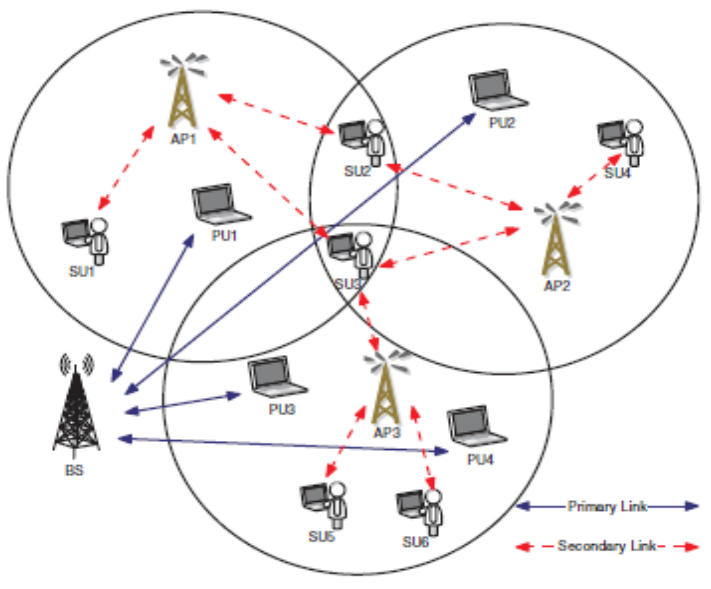

Fig. 1. Scenario of CCRN

The channel conditions are assumed to be stable during a fix time slot $\square$, but vary independently from one slot to another. The spectrum sharing strategy operates in a time-slotted manner and transmission channels are assumed to conform to a Rayleigh flat fading model. The CSI is available, which is estimated by exploiting techniques such as least squares (LS) estimation and minimum mean-square-error (MMSE) estimation

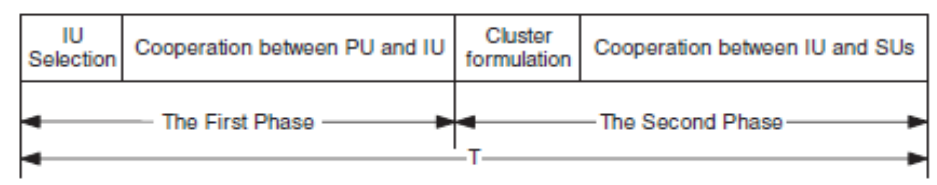

Fig. 2. Time frame structure for the spectrum sharing strategy

The SUs, who participate in the cooperation with the PUs, send feedbacks with their transmit power values they want to devote in delivering PUs' traffic to the BS. In order to improve the performance of primary network, the BS broadcasts the cooperation selection requirement to its surrounding SUs. If one SU can serve as the relay for multiple PUs, it sends different transmit power values corresponding to each PU to the BS. However, in real networks, some SUs might not be willing to cooperate with the PU, as it is quite energy consuming to relay PU's traffic while the utility gain might be relatively low, i.e., the ratio of utility to power consumption is low.

But the SUs still desire to gain the secondary transmission opportunities so as to improve the utility. In order to solve the aforementioned problem, the selected IU cooperates with the remaining SUs to benefit them. Meanwhile, through the cooperation between cluster head IU and other SUs in the cluster, the IU can improve its own performance as well.

As shown in Fig. 2, The time frame structure includes two cooperations: the first phase cooperation and the second phase cooperation. In the IU selection period of the first phase cooperation, after BS acquires the acknowledgement and the information from potential IUs, the BS exploits the maximum weighted bipartite matching algorithm to find the most appropriate cooperative SUs, i.e., the IUs. After partner IU selection, the PU cooperates with the IU in a TDMA manner. Then, the IU broadcasts its cooperation requirement to begin the second phase cooperation.

The SUs send the acknowledgement that they want to join into the cooperation with the IU. After that, the IU transmits its packet towards the associated AP. During this transmission process, the surrounding SUs (form a cluster) who are involved in the cooperation can overhear the data. Then, by using network coding, the SUs in a cluster create new combinations of packets from the received packets and transmit those towards the respective AP. The cooperation scheme among cluster head IU and SUs in the cluster is referred as cooperative network coding, in which the IU is the source and the corresponding AP is the destination, and the SUs form a cluster to help IU relay the data from the source to the destination.

Energy efficiency is considered in the system by using a ratio of utility to energy, which enables a tradeoff between utility and energy consumption. IU selection is performed to select the IUs who cooperate with the PUs. The IUs are a group of SUs that have better channel conditions than other SUs to relay PUs' traffic.

\section{NUMERICAL RESULTS}

In this section, in comparison with the random selection scheme, the IU selection scheme is evaluated in a CCRN simulator. The operation factors, e.g., cooperation time allocation and SUs' power consumption, are also investigated.

As shown in Fig. 1, there are 4 PUs and 6 SUs in the CCRN. The powers of PUs and SUs vary from $1 \mathrm{~mW}$ to $2 \mathrm{~mW}$ and from $0.5 \mathrm{~mW}$ to $1.5 \mathrm{~mW}$, respectively. The proposed IU selection (IS) scheme and random selection (RS) scheme, are compared i.e. the performance obtained by using two different schemes. 


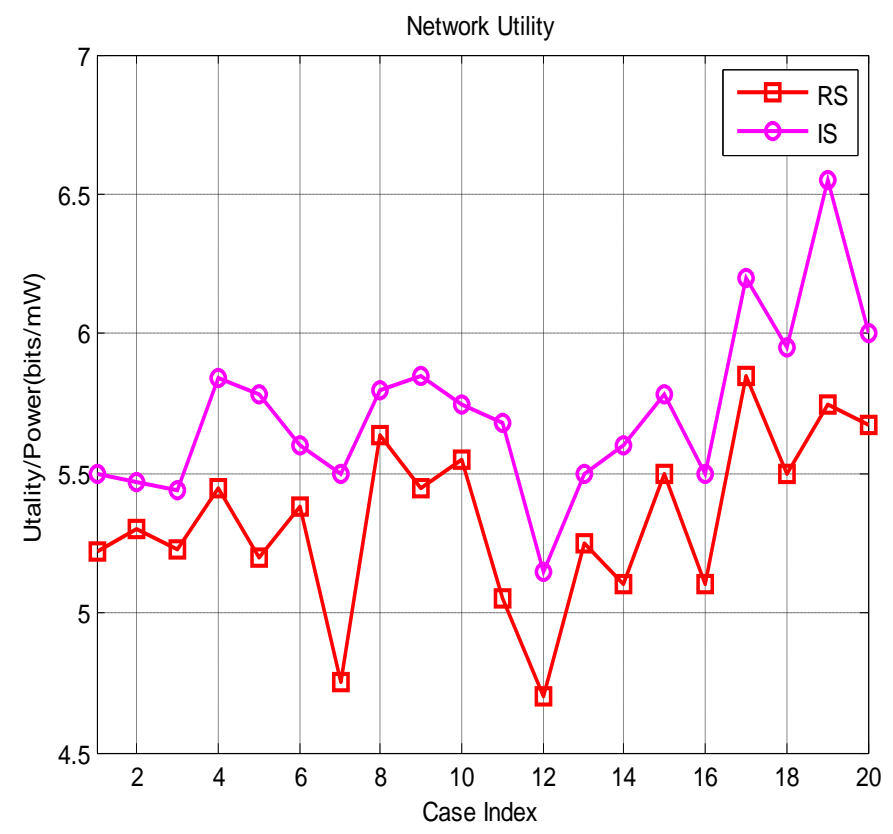

Fig. 3. Comparison of the network utility attained by two different schemes

In Fig. 4 for the BS under different values of IU's power is evaluated, by the impact of choosing the value of $\alpha$. From the candidate SUs in the cooperation Once BS collects the information; BS chooses an appropriate value of $\alpha$ and to select the IUs, performs the maximum weighted matching. The whole utility of cooperation pairs is simulated and the utility for different values of $\alpha$ is demonstrated in the figure.

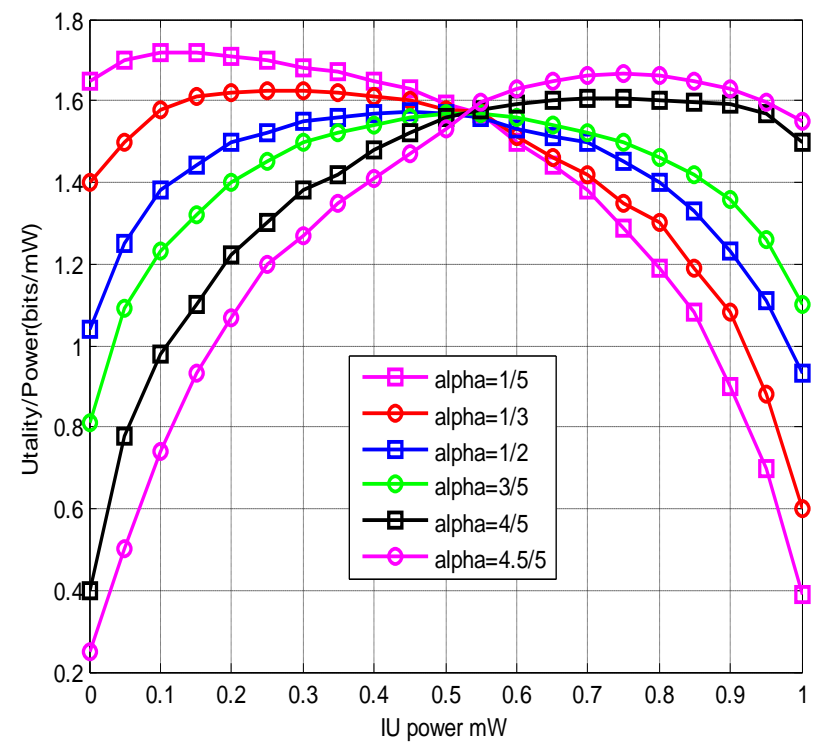

Fig.4. Achieved utility vs. IU's power for different values of $\alpha$.

\section{CONCLUSION}

In this paper, we have studied and implemented a novel cooperative spectrum sharing approach for a wireless network consisting of multiple primary and secondary users. we have seen a spectrum sharing strategy based on two-phase cooperation including an IU selection scheme in CCRN. The cooperation pairs between PUs and IUs have been obtained,
By solving the maximum weighted bipartite matching problem. Thus we have got the maximum total utility.

Further, the energy efficiency have been considered in the IU selection problem and The selected IU cooperates with the PU as well as its surrounding SUs. With the help from the IUs the system utility and the spectrum access opportunity have been improved. With the help of simulated result we have find that the utility obtained by performing the proposed partner IU selection scheme is always higher than that attained by the random selection scheme in our CCRN. In future work, we will analyze the cooperation between the IU and the surrounding SUs in detail.

\section{REFERENCES}

[1] Akyildiz, W. Y. Lee, M. Vuran, and S. Mohanty, "Next Generation/ Dynamic Spectrum Access/Cognitive Radio Wireless Networks: A survey," ACM Journal on Computer Networks, vol. 50, no. 13, pp. 2127- 2159, Sep. 2006.

[2] A. Goldsmith, S. Jafer, I. Maric, and S. Srinivasa, "Breaking spectrum gridlock with cognitive radios: An information theoretic perspective," in Proceedings of IEEE, vol. 97, no. 5, pp. 894-914, May 2009.

[3] O. Simeone, I. Stanojev, S. Savazzi, Y. Bar-Ness, U. Spagnolini, and R. Pickholtz, "Spectrum leasing to cooperating secondary ad hoc networks," IEEE Journal on Selected Areas in Communications, vol. 26, no. 1, pp. 203-213, Jan. 2008.

[4] Y. Han, A. Pandharipande, and S. Ting, "Cooperative decode-and-forward relaying for secondary spectrum access," IEEE Transactions on Wireless Communications, vol. 8, no. 10, pp. 4945-4950, Oct. 2009.

[5] N. Zhang, N. Lu, R. Lu, J. W. Mark, and X. Shen, "Energy-efficient and trust-aware cooperation in cognitive radio networks," in Proceedings of IEEE International Conference on Communications, pp. 1763 1767, Jun. 2012. 
[6] T. Elkourdi and O. Simeone, "Spectrum Leasing via Cooperation with Multilpe Primary Users," IEEE Transactions on Vehicular Technology, vol. 61, no. 2, pp. 820-825, Feb. 2012.

[7] V. Mahinthan, L. Cai, J. W. Mark and, X. Shen, "Partner Selection Based on Optimal Power Allocation in Cooperative-Diversity Systems," IEEE Transactions on Vehicular Technology, vol. 57, no. 1, pp. 511-520, Jan. 2008 .

[8] M. Seyfi, S. Muhaidat, and J. Liang, "Relay Selection in Underlay Cognitive Radio Networks," in Proceedings of IEEE Wireless Communications and Networking Conference, pp. 283-288, Apr. 2012.]
[9] M. Biguesh and A. B. Gershman, "Training-Based MIMO Channel Estimation: A Study of Estimator Tradeoffs and Optimal Training Signals," IEEE Transactions on Signal Processing, vol. 54, no. 3, pp. 328-339,Mar. 2006.

[10] "Spectrum Sharing Strategy using Bipartite Matching for Cooperative Cognitive Radio Networks"Yujie Tang, Yongkang Liu, Jon W. Mark and Xuemin (Sherman) Shen Centre for Wireless Communications, University of Waterloo, ON, Canada, N2L 3G1 Globecom 2013 Cognitive Radio and Networks Symposium 